\title{
Corrigendum: A first step toward understanding patient safety
}

\section{Kyoung Ok Kim}

Department of Anesthesiology and Pain Medicine, Dongguk University Ilsan Hospital, Goyang, Korea

Korean J Anesthesiol 2016 October 69(5): 429-434

In this paper, Acknowledgments section was omitted unintentionally.

The author apologize for any inconvenience this mistake may have caused.

\section{Acknowledgments}

This work was supported by the Dongguk University Research Fund of 2015. 